\title{
Bi objective hybrid vehicle routing problem with alternative paths and reliability
}

\author{
Mahmood Nosratia and Alireza Arshadi Khamseh ${ }^{b^{*}}$
}

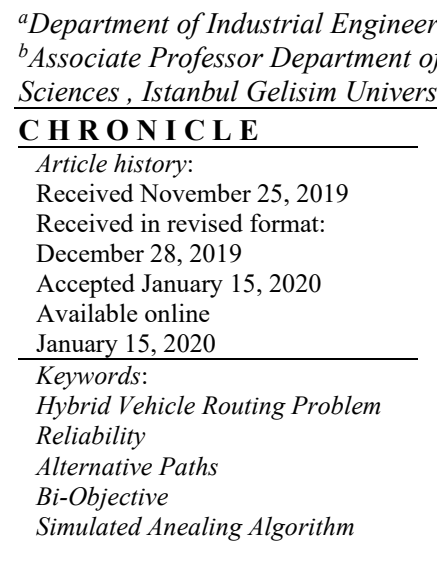

\section{Introduction}

The supply chain (SC) can be considered as a network, which includes suppliers, manufacturers, warehouses, and distribution centers and designed to maximize customers' benefits (Tiwari \& Chang, 2015). Logistics is a division of the SC, which is divided into physical activities such as transportation and warehousing and non-physical activities such as supply chain design and transportation discussions (Tseng et al., 2005). In a simple word, the process of delivering a product (service) in the required quantities, appropriate condition, and within the set time and place at an accepted value is called logistics (Ćirović et al., 2014). In logistics systems, transportation plays a critical role in moving raw materials from suppliers to manufacturers and distributing products from the manufacturers to the customers. In United States, the transportation section accounts for about $28 \%$ of energy expenditure. Therefore, transportation costs have a meaningful impression on the price of final products (Beamon, 1998; Reed et al., 2008, Farahani et al., 2011).

In recent years, the issue of greenhouse gas emissions and their impact on the environment has become a global concern. The European Commission confirmed that one-fifth of total carbon dioxide emissions in the European Union belong to road transportation (Felipe et al., 2014; Demir et al., 2014). In logistics, transportation is one of the most influential aspects and an essential foundation for economic growth. However, it is one of the major consumers of fuel and a large proportion of all pollutants.

* Corresponding author.

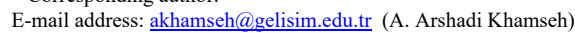

(C) 2020 by the authors; licensee Growing Science, Canada doi: $10.5267 /$ j.dsl.2020.1.002 
Today, many logistics companies are launching green logistics projects to reduce $\mathrm{CO}_{2}$ emissions (Affi et al., 2018). Due to the enormous influence of logistical activities on the environment, issues such as green logistics have been raised in which, along with financial discussions, they also focus on environmental protection issues (Ping, 2009).

Vehicle Routing Problem (VRP) has been recognized as a fundamental optimization problem wherein transportation costs are minimized by planning proper routes for vehicles (Vincent et al., 2017). Lin et al. (2014) surveyed 284 research studies on VRP and categorized them into two major groups: Traditional VRP and Green Vehicle Routing Problem (GVRP). The Green Vehicle Routing problem is a kind of routing problem that contains issues such as vehicles with different fuels and alternative filling stations and adapts environmental and economic consequences (Schneider et al., 2014; Erdoğan \& Miller-Hooks, 2012).

One of the green logistics techniques is to use environmental-friendly vehicles such as hybrid vehicles to reduce greenhouse gas emissions (Erdoğan \& Miller-Hooks, 2012). The hybrid VRP is an extended model of the green VRP in which the vehicles can provide the energy required for their movements in two ways, namely electrical fuel and Traditional fuel. Vehicles can choose and change the implementation of any fuel at any time (Mancini, 2017). This problem focuses on minimizing the total costs while hybrid vehicles are using. Therefore, the hybrid vehicle routing problem classified as green vehicle routing problems by Lin et al. (2014).

This paper studies an extension of HVRP in which alternative paths are considered. Each of the alternative paths has features that can help to improve the quality of the answers. In this problem, two objective functions are developed. The first objective is to minimize the total cost of routing, and the second one is to maximize system reliability. To solve the proposed model, the e-constraint method and meta-heuristic algorithm are used. This paper is prepared as follows. Section 2 presents the literature review on green vehicle routing problem and reliability optimization and metaheuristic algorithm in VRP. Section 3 introduces the problem and assumptions. Mathematical formulations are detailed in section 4 . Section 5 describes solving methods. Computational results are presents in section 6. Lastly, section 7 gives conclusions and further research plans.

\section{Literature review}

The problem studied in this paper scrutinizes a bi-objective green vehicle routing problem with reliability optimization. Accordingly, this section will review vehicle routing problems, reliability optimization, and Appropriate meta-heuristic algorithms.

\subsection{Green vehicle routing problems}

In recent years, green logistics have given much attention and includes the combination of environmental aspects in logistics. Dekker et al. (2012) performed an overview on topics such as intermodal transit, models of transportation modes, fleet operation and selection, intelligent distribution systems and fuel selection. One of the emerging research areas is to minimize the dissemination of pollution. In this regard, Bektash and Laporte (2011) presented a discussion about pollution routing problem. PRP is an extension of the classic Time Windows vehicle routing problem, which includes routing several vehicles to serve a collection of customers and determining the speed of these vehicles in each section of the route to minimize a function including fuel consumption, pollution emission costs, and driving costs. Demir et al. (2012) addressed the same issue. They proposed an innovative approach based on an adaptive large neighborhood search. The time-dependent pollution routing problem was developed by Franceschetti et al. (2013). Furthermore, Demir et al. (2014) introduced the problem of two-level pollution routing. 
Kara et al. (2007) introduced a new cost function based on distance and load of vehicles for capacitated vehicle routing issues, which is referred to as energy-saving issues in vehicle routing. Kuo (2010) introduced a model for determining total fuel consumption in a time-dependent vehicle routing problem, in which speed and trips time depend on the duration of the travel when planning the route. Xiao et al. (2012) presented a mathematical optimization model to achieve fuel consumption rates, in which vehicles with limited capacity were considered. Few articles on VRP have dealt with alternative fuel vehicles. In a paper, Gonçalves et al. (2011) studied a pickup and delivery vehicle routing problem with multiple fleets where vehicles used both traditional and electrical fuel types. The goal of this paper was to minimize total costs, including fixed and variable costs of the vehicle. They took into account time and capacity constraints and added extra time to charge the batteries of electric vehicles. However, they did not explicitly consider the location of the recharging station in their model.

An article by Conrad and Figliozzi (2011) raised the issue of recharging vehicles in the VRP, in which vehicles were allowed to refuel directly at customer sites and add extra time as a penalty to the total route time. Erdoğan and Miller-Hooks (2012) were the first to point out the VRP with refueling stations. They developed techniques to help transportation overcome the problems that result from limited refueling infrastructure. These techniques plan for stopping and refueling at refueling stations to eliminate the risk of finishing the fuel while maintaining low-cost paths. Schneider et al. (2014) presented the electric vehicles routing problem with time constraints and refueling stations, which can consider as a type of GVRP. Bruglieri et al. (2015) made an extension of this issue. As such, partially refueling allowed in the problem. Philip et al. (2014), in an article, presented the technology of multiple recharging with various recharging time and cost.

Vincent et al. (2017) presented a hybrid vehicle routing problem, which is an extension of the green VRP. This paper focused on vehicles that use hybrid energy sources. A mathematical model with the aim of minimizing the total cost was developed. According to this model, it is possible to use both types of traditional and electrical fuel due to the availability of electric charging centers and refueling stations. Mancini (2017) introduced and formulated a hybrid VRP in an article. The new problem is an extended model of VRP in which the vehicles can provide two types of energy needed for their movement: 1 . Traditional fuel, 2. Electric fuel. Vehicles can select and change the use of any fuel at any time. When using electric fuel, the vehicles' travel cost is lower, but the electric batteries have a limited capacity and need to recharge at recharging stations. The number of recharging stations is also limited. Each time the battery discharged, the vehicle automatically uses traditional fuels to continue its journey.

In many routing problems, only the shortest path is considered, but in some circumstances, the quality of the responses obtained can be affected by several alternatives to cross the first node to the end node. In a paper, Garaix et al. (2010) studied the case where there were alternatives paths between every two paths, In this case, the shortest route may have high traffic and disruption, while the longer routes may reduce time and fuel consumption.

\subsection{Reliability optimization}

Nowadays, risk management in supply chain and distribution systems is considered by using reliability. Nishiguchi and Beaudet (2008), through a case study in Toyota manufacturing failure caused by suppliers' problems, explained that the existence of a close relationship with one supplier rather than a low relationship with several suppliers has more impact on increasing the reliability. Liu et al. (2010) argued that the reliability of each component limits the maximum reliability of the total supply chain. They pointed to reliability and its importance in the supply chain, but they did not provide any mathematical definitions or models. Klimov and Merkuryev (2008) defined supply chain reliability based on lifetime and presented a method for evaluating supply chain reliability using structural reliability theory. In an article, Zaitsev (2012) defined reliability as the probability of the correct operation of a product for a specific period, which can replace the supply chain risk. He also argued 
that strategies could be developed to analyze the risks of supply chains quantitatively and avoid significant failures using reliability engineering.

Instead of mathematical definitions, Adenso-Diaz et al. (2012) have provided a literal definition of supply chain reliability: The probability that all materials and products will reach their destinations via the network within a specified time and under stated conditions. They also investigated the impact of thirteen supply chain characteristics such as system density and complexity and component reliability on supply chain reliability through simulation. Ha et al. (2018) proposed the brief and mathematical definition of supply chain reliability and described related functions such as risk, cumulative risk, and availability at the level of production components. Besides, they provided structural reliability models such as series, parallel, parallel-series, and series-parallel, using reliability theory in system-level so that supply chain reliability is in line with the supply chain's base activities.

\subsection{Using the meta-heuristics algorithm in the vehicle routing problem}

Using meta-heuristic algorithms as a general solver for optimization problems requires extensive adaptation and user intervention or expertise to match the algorithm and the properties of each problem. The vehicle routing problem is one of the significant combinatorial optimization problems with a wide range of applications and is an excellent case for applying the meta-heuristic approach expressed by Gendreau et al. (2001) and Laporte (2009). These algorithms are subdivided into neighborhood-based, population-based, and hybrid approaches (Vidal et al., 2013).

Neighborhood-based algorithms usually move to a single optimal solution with iterative neighbor searches such as simulated annealing (Kirkpatrick \& Vecchi, 1983; Osman, 1993), tabu search (Glover \& Laguna, 2013; Taillard, 1993; Rochat \& Taillard, 1995), variable neighborhood search (Mladenović \& Hansen, 1997; Kytöjoki et al., 2007; Chen et al., 2010; Affi et al., 2018), an adaptive large neighborhood search (Ropke \& Pisinger, 2006), and iterated local search (Lourenço et al., 2003). Population-based approaches are often inspired by natural mechanisms and provide one or more new solutions by existing compounds. The most well-known of these algorithms are genetics and Evolutionary Algorithms (Holland, 1975), Memetic Algorithms (Moscato, 1989; Moscato \& Cotta, 2010), Path relinking, and Scatter search (Glover, 1999; Resende et al., 2010), Particle Swarm Optimization (Eberhart \& Kennedy, 1995; Poli, 2007) and the ant colony optimization (Dorigo \& Stützle, 2003). Hybrid algorithms combine different methods to use more power in solving. Various types of these methods have been proposed, for example, simulated annealing and Tabu Search (Osman, 1993), Tabu Search and iterated local search (Cordeau \& Maischberger, 2012), and large neighborhood and variable neighborhood search (Rincon-Garcia et al., 2017).

The simulated annealing (SA) algorithm as an extension of the Markov Chain Monte Carlo method was first introduced by Metropolis et al. (1953). The SA algorithm is inspired by the solid metals annealing process. Subsequently, SA has become a popular meta-heuristic algorithm for solving optimization problems. Recent advances in meta-heuristic algorithms have shown that various modifications can be made to improve the performance of these algorithms, such as: Using a fuzzy system to determine the probability of accepting worse solutions or many other parameters in metaheuristic algorithms (Avila \& Valdez, 2015; Castillo et al., 2015). Osman (1993) and Lin et al. (2009) have proposed hybrid simulated annealing that leads to higher quality solutions. Vincent et al. (2010) presented a straightforward and authoritative version of SA that incorporates diversity in response neighborhoods. This version has been successfully implemented in a variety of VRPs, such as truck and trailer routing problems (Lin et al., 2011), team orienteering problem with time windows (Lin et al., 2012), and open vehicle routing problem with cross-docking (Vincent et al., 2016).

Although most VRP research papers focus on single-objective optimization, today, the use of multiobjective optimization is of interest to researchers because it offers new opportunities to define 
problems. Some multi-objective VRPs are solved using cumulative approaches where all objective functions are incorporated into a single function using a combination of mathematical operations. The bug of such an approach is the accurate determination of weights, especially when there are insufficient information and knowledge on widespread routing problems in the real world (Tan et al., 2006). Hence approaches, that are offering a wide range of solutions, representing a balance between goals, and as well as Pareto-based strategies are more appropriate for multi-objective optimization (Goldberg, 1982).

Jozefowiez et al. (2008) categorized the different goals of various types of VRP according to the components that it is associated with paths, resources, and so on. The most usually used common goals are such as cost reduction (distance and time), path length reduction, and path balance. Also, they provide two strategies of scalar (weighted aggregation) strategies and evolutionary algorithms to solve multi-objective routing problems that most preferred by researchers. The multi-objective evolutionary algorithm has been used in the works of Ombuki et al. (2006), Jozefowiez et al. (2009), and GarciaNajera and Bullinaria (2011). The other multi-objective version based on meta-heuristic methods have also been used in VRP, such as particle swarm by Norouzi et al. (2009), Pareto ant colony by Chávez et al. (2016), and hybrid algorithm by Baños et al. (2013) wherein they combined evolutionary computation and simulated annealing.

\section{Problem statement}

HVRP involves meeting a set of clients by vehicles. Every vehicle must start its journey from the depot and return to it again. In this problem, The fleet composed of identical vehicles. For each point in the route, the service time is considered. Each pair of customers has travel time and distance. Travel speeds on any route are considered to be constant. Vehicles must serve the assigned customers on each tour at the indicated time. Any vehicle can refuel along the way. When refueling, it is assumed that the fuel tanks are filled to the maximum capacity. There is no limit on the number of refueling stops. Refueling stations may be used many times by the fleet. For this purpose, dummy duplicates are assumed for each station, which must be visited at most once. The number of these dummy duplicates is a crucial problem (Mancini, 2017). If too many copies are added, the dimensions of the network become too high, and the model becomes inefficient, while if too few copies are added, the solutions obtained may not be optimal.

In this problem, there are different paths between two points. Considering different paths between each source and destination has different results. In some cases where only is examined the shortest route, different alternatives will only increase the response search space, But in many cases, in terms of some factors, a route except the shortest route has priority, which may increase the quality of the answers. Factors such as traffic, natural disasters, problems with electricity or water, and sewage can disrupt a route and make the route unusable. In general, reliability in systems can be defined as the ability of the system or subsystem to perform specified and predefined missions correctly, under specified conditions, and over a specified time, which usually expressed as several probable parameters. Therefore for each route, we can consider reliability, which means the probability of the route usability within a specified time.

\subsection{Model assumptions}

- This model is definite and static.

- The hybrid routing problem involves customers, a warehouse and a set of refueling stations.

- Each time the device returns to the warehouse, the capacity of the fuel tanks is filled to the maximum.

- All stations have unlimited capacities and are always capable of servicing vehicles, and there is no limit to the number of stops at refueling stations for vehicles. 
- Each time a vehicle meets a refueling station, the capacity of the vehicle's fuel tanks is filled to the maximum.

- Each refueling station is allowed not to meet more than once.

- Vehicles are needed to meet all customers.

- Vehicle can meet refueling stations when needed to find the lowest cost of the tour.

- The cost of fuel consumption are different.

- There are alternative routes with different distances, travel time, and reliability between the two points of the route.

\section{Mathematical formulation}

The hybrid vehicle routing problem, according to the work of Erdoğan and Miller Hook (2012), has been developed as follows. This problem is defined on a complete unidirectional graph of $G=(V, E)$ such that $\mathrm{V}$ is a set of $\mathrm{I}=\left\{v_{1}, v_{2}, \ldots, v_{n}\right\}$ customers, $v_{0}$ warehouse, and $\mathrm{F}$ is a set of $\mathrm{f}$ refueling stations $\mathrm{F}=\left\{v_{n+1}, v_{n+2}, \ldots, v_{n+f}\right\}$. The entire set of vertices is $V=\left\{v_{0}\right\} \cup I \cup F=\left\{v_{0}, v_{1}, v_{2}, \ldots, v_{n+s}\right\}$. It is assumed that in addition to refueling stations, the depot can also be used as a refueling station, and all refueling stations have unlimited capacities. The set of $\mathrm{E}=\left\{\left(v_{i}, v_{j}\right): v_{i}, v_{j} \in V, i<j\right\}$ corresponds to the edges connecting the vertices of $\mathrm{V}$.

$\begin{array}{cl}\text { Index } & \\ I & \text { Customers } \\ I_{0} & \text { Customers and warehouses } \\ F & \text { Refueling stations } \\ F_{0} & \text { Refueling stations and warehouses } \\ V & \text { Warehouses, customers and refueling stations } \\ K & \text { Fuel types } \\ S & \text { Different paths }\end{array}$

Parameters

$m \quad$ Number of allowed vehicles

$r \quad$ Fuel consumption rate

$Q_{k} \quad$ The capacity of fuel type k tank

$P \quad$ Service time

$T_{\max } \quad$ Maximum allowed time for each route

$d_{i j s} \quad$ The distance of path $\mathrm{S}$ between node $\mathrm{i}$ and $\mathrm{j}$

$t_{i j s} \quad$ Interval time of path $\mathrm{S}$ between node $\mathrm{i}$ and $\mathrm{j}$

$R_{i j s} \quad$ Reliability of path $\mathrm{S}$ between node $\mathrm{i}$ and $\mathrm{j}$

$c_{k} \quad$ Cost of fuel type $\mathrm{k}$

Decision variables

$y_{j k} \quad$ level of fuel type $\mathrm{k}$ tank at node $\mathrm{j}$, This amount adjusts to $\mathrm{Q}$ at the refueling stations and depot.

$\tau_{j} \quad$ The vehicle arrival time to node $\mathrm{j}$, This value is zero at the exit of the warehouse

Binary decision variables

$x_{i j} \quad$ Equal to 1 if the vehicle travels between $\mathrm{i}$ and $\mathrm{j}$; otherwise it is zero

$x_{i j k s}^{\prime} \quad$ Equal to 1 if the vehicle with fuel type $\mathrm{k}$ travels between $\mathrm{i}$ and $\mathrm{j}$ from path $\mathrm{S}$;

Mathematical model 


$$
\begin{aligned}
& \min _{1}=\sum_{\substack{i \in V \\
i \neq j}} \sum_{\substack{j \in V \\
j \neq i}} \sum_{k} \sum_{s} d_{i j s} x_{i j k s}^{\prime} c_{k} \\
& \max _{2}=\prod_{i} \prod_{j} \prod_{k} \prod_{s}\left(1-\left(\left(1-R_{i j s}\right) * x_{i j k s}^{\prime}\right)\right)
\end{aligned}
$$

s.t

$$
\begin{aligned}
& \sum_{j \in V} x_{i j}=1 \quad \forall i \in I \\
& \sum_{j \in V}^{j \neq i} x_{i j} \leq 1 \quad \forall i \in F \\
& \sum_{i \in V}^{j \neq i} x_{j i}-\sum_{i \in V} x_{j i}=0 \quad \forall j \in V \\
& \sum_{j \in V}^{j \neq i} x_{0 j} \leq m \\
& \sum_{\substack{j \in V \\
j \neq 0}}^{j \neq 0} x_{j 0} \leq m \\
& \tau_{j} \geq \tau_{i}+\left(\sum_{k} \sum_{s}\left(t_{i j s}+p\right) \cdot x_{i j k s}^{\prime}\right)-T_{\max }\left(1-x_{i j}\right) \quad \forall i \in V, j \in V \backslash\{0\} \text { and } i \neq j \\
& 0 \leq \tau_{0} \leq T_{\max } \\
& t_{0 j s} \leq \tau_{j} \leq T_{\max }-\left(t_{j 0 s}+p\right) \quad \forall s, j \in V \backslash\{0\} \\
& y_{j k} \leq y_{i k}-r \cdot\left(\sum_{k} \sum_{s}\left(d_{i j s} * x_{i j k s}^{\prime}\right)\right)+Q_{k}\left(1-x_{i j}\right) \quad \forall j \in I, i \in V, k \text { and } i \neq j \\
& y_{j k}=Q_{k} \quad \forall k, j \in F_{0} \\
& y_{j k} \geq \min \left\{r \cdot\left(\sum_{s}\left(d_{j 0 s} * x_{j 0 k s}^{\prime}\right)\right), r .\left(\sum_{s}\left(d_{j i s} * x_{k s}^{\prime}\right)+\sum_{s}\left(d_{i 0 s} * x_{i 0 k s}^{\prime}\right)\right)\right\} \\
& \forall k, s, j \in I, i \in F \\
& \sum_{k} \sum_{s} x_{i j k s}^{\prime}=x_{i j} \quad \forall i, j \text { and } i \neq j \\
& x_{i j}, x_{i j k s}^{\prime} \in\{0,1\} \quad \forall i, j, k, s
\end{aligned}
$$

The first objective function (1) is related to minimize the total cost. The second objective function (2) is proposed to maximize the total reliability of the system. To calculat it, considering the nature of the routing problem and relation of routes to each other, the routes are considered in series. Constraint (3) states that each customer is met exactly once. Constraint (4) states that each refueling stations may be met just once. By constraint (5), route continuity is ensured. Constraints (6) and (7) specify the maximum number of routes or vehicles. The time to reach each point is traced by the constraint (8). 
Constraints (9) and (10) ensure that any vehicle does not return to the warehouse later than the indicated time. The fuel level of vehicles at any point is guaranteed by the constraint (11).

Constraints (8) and (11), in addition to being time and fuel constraints, guarantee that the sub tours do not create. Constraint (12) makes the fuel level equal to $\mathrm{Q}$ when leaving the warehouse and refueling centers. Constraint (13) ensures that the remaining fuel is sufficient to reach the depot or refueling stations. Constraint (14) specifies that movement between two points must take place from a given path and a specified fuel. Finally, constraint (15) shows the domain of variables.

\section{Solving methods}

In this paper, two methods for optimizing the model are discussed. The first one is the exact method which solves the model using the epsilon constraint method. The second approach is to solve the problem using the MOSA meta-heuristic algorithm. In this method, which is coded in MATLAB, we try to find the optimal solutions close to the exact method. This solution can be helpful for larger size models. The solutions obtained by this method are not necessarily optimizable.

As the VRP is known as NP-hard (Problems that in it, computations increase exponentially by increasing the size of the problem), the GVRP and presented model, as particular cases of the VRP, are also in this category. As a result, when the dimensions of the problem become larger, it is not possible to solve the problem in a reasonable time, or it is costly (Erdoğan \& Miller-Hooks, 2012). For this reason, it is essential to use the meta-heuristic approach to obtain accurate answers at reasonable times. According to the review of the literature and similar research, the MOSA algorithm is one of the most effective methods to solve this kind of problem.

\subsection{Epsilon constraint method}

The epsilon constraint method is one of the most popular methods of solving multi-objective optimization problems (Ehrgott \& Gandibleux, 2002). In this way, instead of combining the objective functions into one function, the optimization of one objective is considered, and other objective functions become constraints called epsilons. The Pareto front can be created by using this method (Bérubé et al., 2009).

\subsection{Multi-objective simulated annealing algorithm}

The first multi-objective version of the simulated annealing algorithm was proposed by Serafini (1994), using a method almost similar to the single-objective SA algorithm. Ulungu et al. (1999) ntroduced multi-objective simulated annealing (MOSA) tested on multi-objective hybrid optimization problems. Their algorithm worked with only one current solution but saved the non-dominated solutions during the search. Nam and Park (2000), presented one of the complete MOSA algorithms. In this paper, a similar version is used.

MOSA uses the concept of dominance and annealing for efficient search. SA's main obstacle to multiobjective optimization is its inability to find multiple solutions. However, SA can do the same thing by repeating the experiments, because the algorithm converges to the global optimum with a uniform probability distribution in single-objective optimizion. It is determined that SA can find each optimum with probability of 0.5 when there are two global optimal (Mitra et al., 1986). The use of SA in multiobjective optimization has advantages over other evolutionary algorithms because it does not require large memory to maintain population and does not use additional algorithms to extend solutions to the Pareto front. Besides, MOSA can find a small group of Pareto solutions in a short time and then find more solutions by repeating experiments for detailed information on the Pareto Front (Nam \& Park, 2000). 


\section{Computational results}

The proposed mathematical model is nonlinear mixed-integer programming that GAMS version 24along with SCIP solver was used to solve it. The proposed meta-heuristic algorithm was also coded in version 2016 of MATLAB. The software was ran on a system with $1.3 \mathrm{GHz}$ Intel Core i5 processor with 8GB of RAM and Windows 64-bit. To evaluate the accuracy and efficiency of the proposed metaheuristic algorithm, comparing the results of this algorithm with the exact ones obtained from the GAMS is necessary. However, this comparison is made in small sizes where the software can achieve optimal results.

\subsection{Numerical examples}

To show the effectiveness of the proposed model, a particular problem in Green Vehicle Routing (GVRP) has been used and the results of which found in the literature. This problem was introduced by Erdoğan and Miller Hook. For this purpose, a network with dimensions of 300 by 330 miles was considered. A warehouse or distribution center was assumed near the center in all cases. Three refueling centers are considered between the distribution center and the grid boundaries in the north, west, and southeast directions. Customers and refueling centers are distributed uniformly across the network (Erdoğan \& Miller-Hooks, 2012).

The capacity of each vehicle's fuel tanks is equal to 60 , and the fuel consumption rate is 0.2 . The total allowed time of each tour is 11 hours, and the service time of each customer or refueling station is 20 minutes. There are two alternative paths between the two nodes in the network in addition to the main path, one ten percent longer and the other ten percent shorter. The reliability of each path is created randomly using a uniform distribution between 0.6 up to 0.99 so that longer routes have more reliability. A template used to name each model. For example, 14C3SU10, where the first number represents the number of customers, the second number indicates the number of refueling centers, and the last number indicates the example number.

\subsection{Performance metrics}

In this article, the following four performance metrics are used as tools for comparison of answers:

Number of non-dominated solutions (NNDS): This metric is the first that is used to compare the set of answers. This metric, regardless of the quality and variety of responses, only counts the number of Pareto answers. Here the better way has more Pareto answers.

Maximum Spread (MS): This metric is one of the criteria for measuring the diversity of Pareto answers introduced by Zitzler (1999), wherein the scatter of answers and the extent of coverage of the Pareto answers are measured. This criterion has applied to two-objective problems and measures the distance between the two borders of the Pareto set. In other words, this index identifies the two endpoints of the set of non-dominated solutions and measures the distance between them.

$$
\mathrm{D}=\sqrt{\sum_{j=1}^{m}\left(\max _{i} f_{i}^{j}-\min _{i} f_{i}^{j}\right)^{2}}
$$

In Eq. (16), $i$ is the index of obtained non-dominated solutions (Pareto), $j$ represents the number of available objective functions, and $f_{i}^{j}$ represents the Pareto answer i-th of the objective function $j$. The higher value of this metric shows a more desirable answer. 
Spacing (S): This metric, along with the Maximum Spread, is the most widely used metrics of dispersion evaluation. In this index, the standard deviation of the consecutive Pareto answers is calculated. The lower value of this standard deviation means that the solutions are reasonably spaced, and the aggregation of answers in a particular area is low, so the low value of this index is a positive point for any algorithm (Schott, 1995).

$$
\mathrm{s}=\sqrt{\frac{1}{n} \sum_{i=1}^{n}\left(d_{i}-\bar{d}\right)^{2}}
$$

In Eq. (17), $d_{i}$ is the Euclidean distance between non-dominated answers, $\bar{d}$ is mean of $d_{i}$, and $n$ is their number.

Mean ideal distance (MID): This metric measures distance between the set of non-dominated answers and an ideal point. As its name implies, the ideal point is unattainable, and in this study, due to objectives of the model, 1 for reliability objective function and 0 for cost objective function assume as the ideal point.

$$
M I D=\frac{\sum_{i=1}^{n} \sqrt{\left(f_{1 i}-f_{1}^{*}\right)^{2}+\left(f_{2 i}-f_{2}^{*}\right)^{2}}}{n}
$$

In Eq. (18), $n$ represents the number of non-dominated answers. $f_{1 i}$ and $f_{2 i}$ represent the values of the first and second objective functions of the $\mathrm{i}$-th non-dominated solution. Also, $f_{1}^{*}$ and $f_{2}^{*}$ are ideal values of objective functions.

\subsection{Parameter setting}

In meta-heuristic algorithms, it is necessary to adjust the parameters correctly to solve the problem desirably. The parameters of the MOSA algorithm are initial temperature $\left(t_{0}\right)$, cooling rate $(\alpha)$, and the

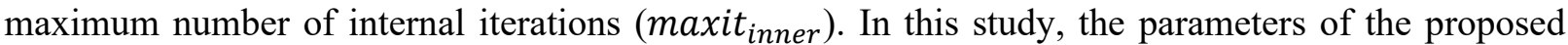
algorithm adjusted by the Taguchi method. This method determines the best possible combination of the input parameters of the algorithm. The results of the MOSA algorithm parameter adjustment by Taguchi shown in Table 1.

\section{Table 1}

Adjusted values of the MOSA algorithm parameters

\begin{tabular}{cccc}
\hline maxit $_{\text {inner }}$ & $\alpha$ & $t_{0}$ & parameter \\
\hline 25 & 0.95 & 10 & Set value \\
\hline
\end{tabular}

\subsection{Results comparisons}

In order to analyze the model and demonstrate the proposed algorithm efficiency, the samples are solved, and the performance metrics of each method are presented in the following tables. Each sample is solved using both exact and meta-heuristic methods. However, as stated, Given that this problem is NP-hard, GAMS is not able to solve large-scale problems in an acceptable time, so the small-scale examples that GAMS can solve them, individually are reported in the Table 2, and two solutions are compared. In this comparison, the execution time of the meta-heuristic algorithm is assumed to be 100 seconds. It is noteworthy that the average GAMS solving time for the first four examples were 3 hours, and the second four examples were approximately 7 hours. 
Table 2

Results and metrics values of GAMS and MOSA algorithm

\begin{tabular}{|c|c|c|c|c|c|c|c|c|c|}
\hline examples & $\begin{array}{l}\text { Solution } \\
\text { Method }\end{array}$ & 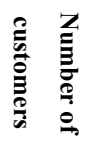 & 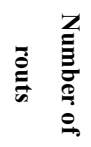 & lower bound & upper bound & NNDS & MS & $\mathbf{S}$ & MID \\
\hline \multirow{2}{*}{ 3C3SU1 } & Gams & 3 & 2 & $(569.71,0.2872)$ & $(683.65,0.8555)$ & 9 & 0.7740 & 0.0215 & 1.0518 \\
\hline & MOSA & 3 & 2 & $(620.76,0.2505)$ & $(735.08,0.798)$ & 10 & 0.7469 & 0.0533 & 1.1518 \\
\hline \multirow{2}{*}{$3 \mathrm{C} 3 \mathrm{SU} 2$} & Gams & 3 & 2 & $(413.28,0.2655)$ & $(677.77,0.8111)$ & 9 & 0.8394 & 0.0679 & 0.9723 \\
\hline & MOSA & 3 & 2 & $(413.28,0.3772)$ & $(583.76,0.8108)$ & 15 & 0.6418 & 0.0536 & 0.9211 \\
\hline \multirow{2}{*}{$3 \mathrm{C} 3 \mathrm{SU} 3$} & Gams & 3 & 2 & $(569.71,0.2872)$ & $(666.53,0.786)$ & 9 & 0.6686 & 0.0237 & 1.0704 \\
\hline & MOSA & 3 & 2 & $(610.97,0.2386)$ & $(724.94,0.798)$ & 10 & 0.7514 & 0.0652 & 1.1425 \\
\hline \multirow{2}{*}{$3 \mathrm{C} 3 \mathrm{SU} 4$} & Gams & 3 & 2 & $(647.47,0.2303)$ & $(837.72,0.7889)$ & 10 & 0.7605 & 0.0212 & 1.1017 \\
\hline & MOSA & 3 & 2 & $(663.91,0.2978)$ & $(816.9,0.6303)$ & 16 & 0.4691 & 0.0258 & 1.1052 \\
\hline \multirow{2}{*}{$5 \mathrm{C} 3 \mathrm{SU} 1$} & Gams & 5 & 2 & $(929.86,0.0502)$ & $(1584.76,0.5240)$ & 8 & 0.6204 & 0.0942 & 1.0547 \\
\hline & MOSA & 5 & 2 & $(1032.56,0.04518)$ & $(1759.83,0.5411)$ & 10 & 0.6639 & 0.0563 & 1.0933 \\
\hline \multirow{2}{*}{$5 \mathrm{C} 3 \mathrm{SU} 2$} & Gams & 5 & 3 & $(781.16,0.118)$ & $(1360.11,0.523)$ & 8 & 0.5937 & 0.0785 & 1.0484 \\
\hline & MOSA & 5 & 3 & $(991.29,0.2114)$ & $(1486.13,0.5739)$ & 9 & 0.5291 & 0.0421 & 1.0630 \\
\hline \multirow{2}{*}{$5 \mathrm{C} 3 \mathrm{SU} 3$} & Gams & 5 & 2 & $(664.67,0.1439)$ & $(958.31,0.623)$ & 8 & 0.6382 & 0.0557 & 1.0910 \\
\hline & MOSA & 5 & 2 & $(795.69,0.2435)$ & $(938.83,0.7018)$ & 9 & 0.5558 & 0.0584 & 1.1488 \\
\hline \multirow{2}{*}{$5 \mathrm{C} 3 \mathrm{SU} 4$} & Gams & 5 & 2 & $(677.49,0.1318)$ & $(1369.23,0.484)$ & 7 & 0.6395 & 0.0535 & 1.0324 \\
\hline & MOSA & 5 & 2 & $(803.67,0.1624)$ & $(1400.32,0.5609)$ & 9 & 0.6265 & 0.0555 & 1.0016 \\
\hline
\end{tabular}

To comparing the solutions, the Pareto front of the results of GAMS and MOSA methods for eight examples are shown in Figs. (1-8). In these graphs, the vertical axis corresponds to the reliability objective function, and the horizontal axis indicates the cost function. As is evident, by increasing costs and using more reliable routes, reliability and total costs rise. Therefore, decision-makers can choose the answer according to the level of reliability and acceptable cost.

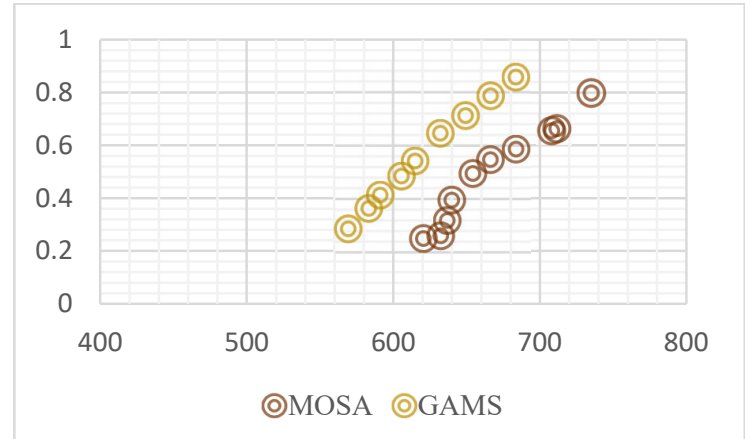

Fig. 1. the Pareto front of Example 3C3SU1

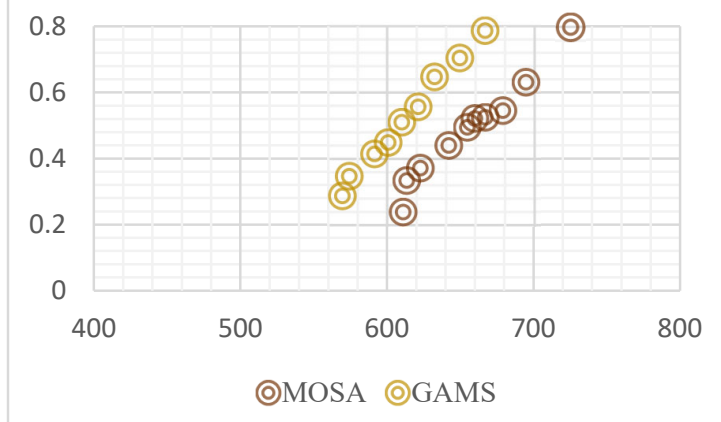

Fig. 3. the Pareto front of Example 3C3SU3

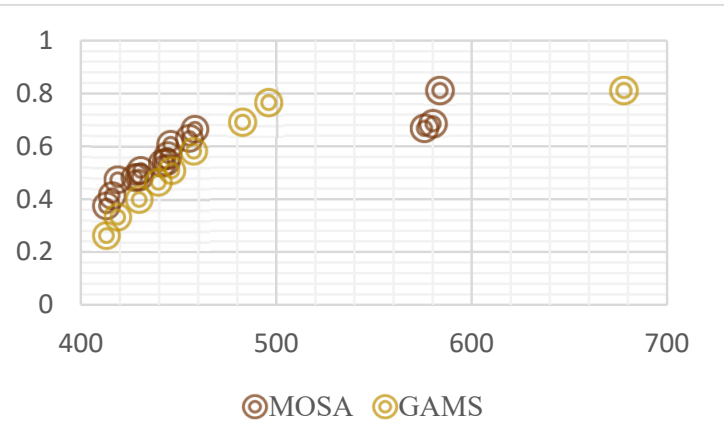

Fig. 2. the Pareto front of Example 3C3SU2

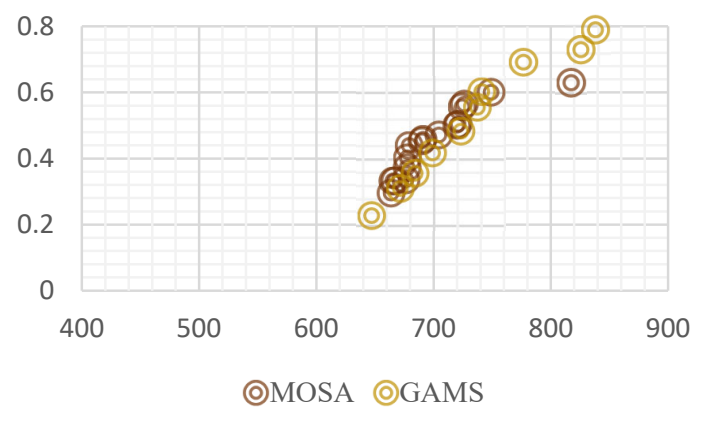

Fig. 4. the Pareto front of Example 3C3SU4 


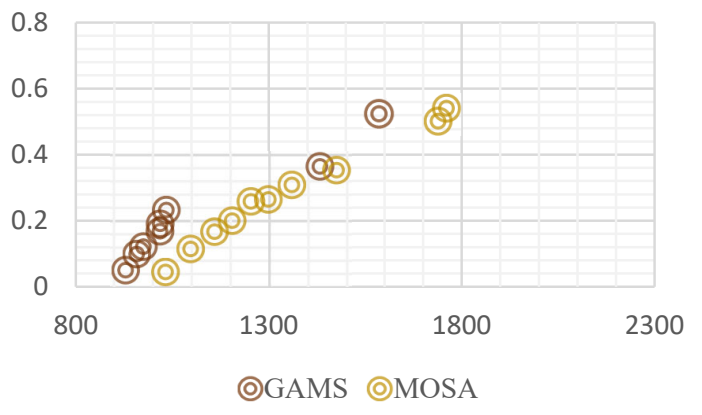

Fig. 5. the Pareto front of Example 5C3SU1

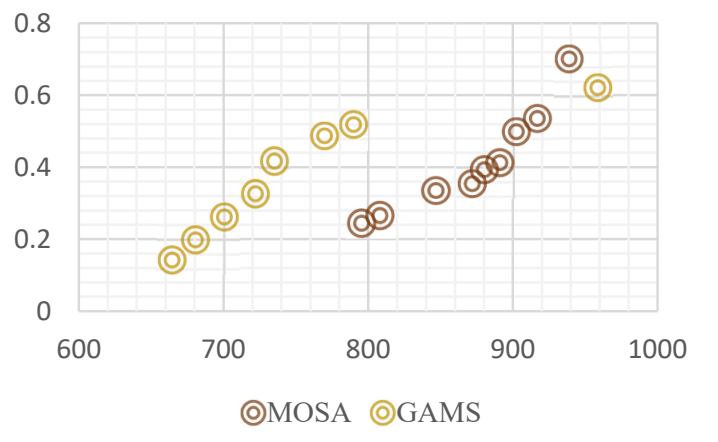

Fig. 7. the Pareto front of Example 5C3SU4

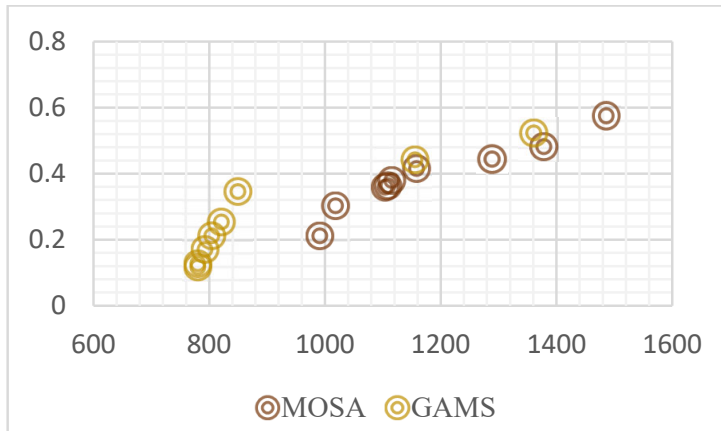

Fig. 6. the Pareto front of Example 5C3SU2

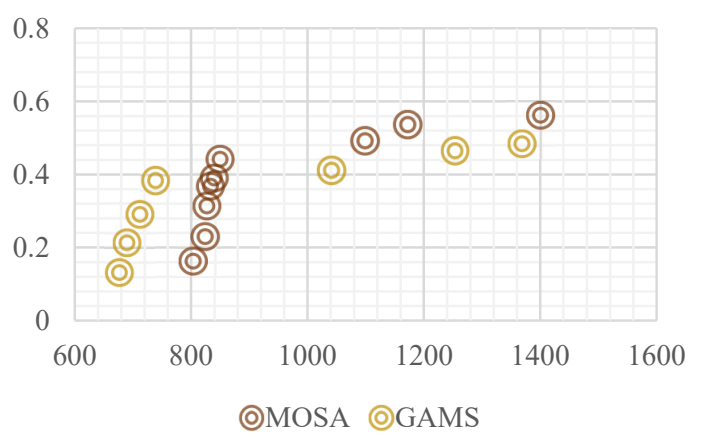

Fig. 8. the Pareto front of Example 5C3SU4

To better compar two methods, Fig. 9 is presented. Based on the number of non-dominated solutions metric, the MOSA algorithm has an advantage over the GAMS method. In the other three indices also, the ability of this algorithm is evident.
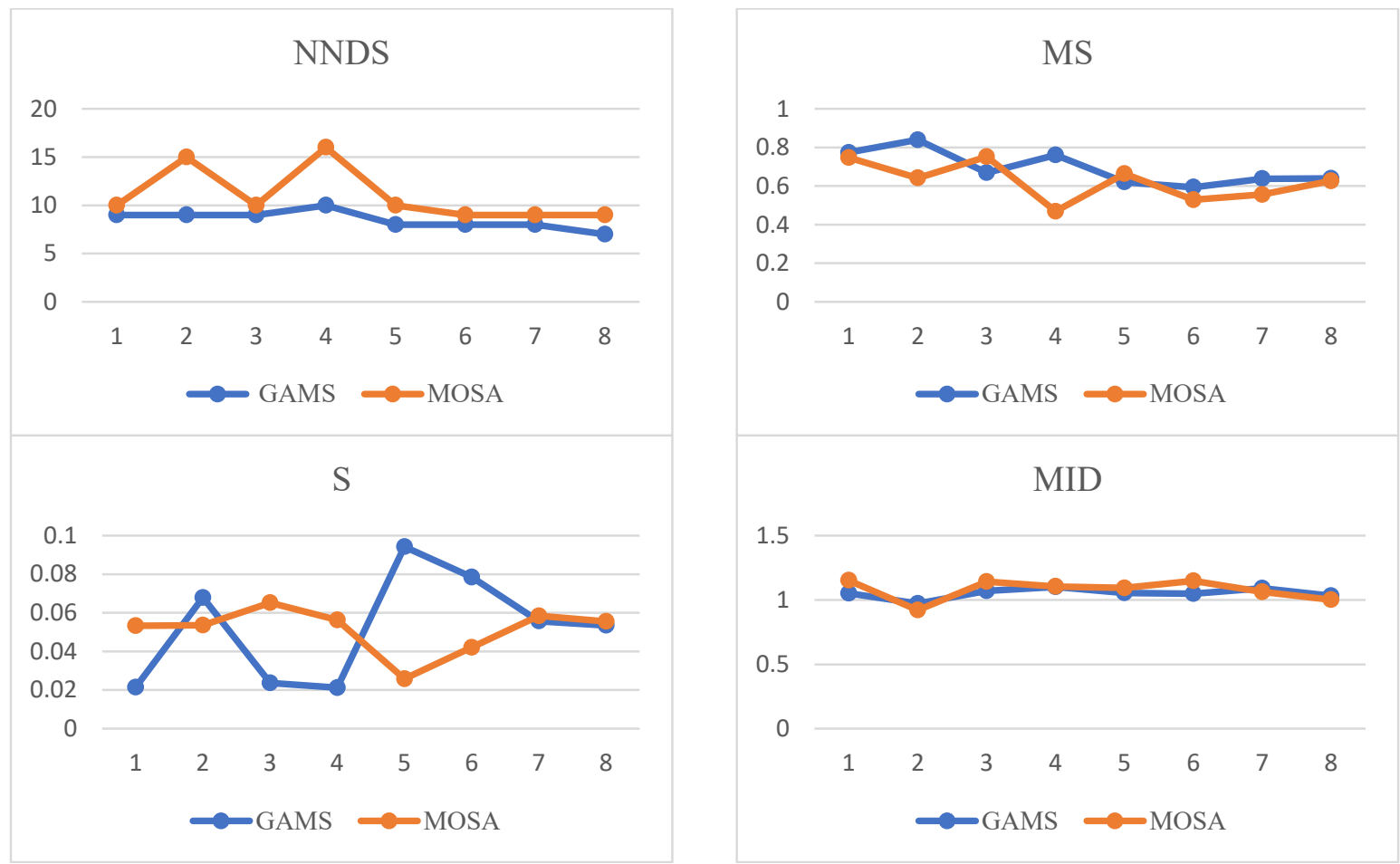

Fig. 9. comparison of solving methods metrics 
Table 3 shows the results of the solutions and performance metrics for samples that GAMS could not solve in a reasonable time, so only the MOSA algorithm was used to solve them. The time taken to solve these problems is 500 seconds.

Table 3

Results and metrics Values of MOSA Algorithm

\begin{tabular}{|c|c|c|c|c|c|c|c|c|}
\hline examples & 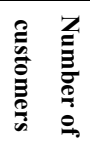 & 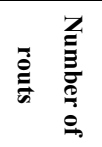 & lower bound & upper bound & NNDS & MS & $\mathbf{S}$ & MID \\
\hline 8C3SU1 & 8 & 3 & $(1659.85,0.1226)$ & $(4522.91,0.4081)$ & 10 & 1.0557 & 0.1199 & 0.8206 \\
\hline 8C3SU2 & 8 & 2 & $(1162.15,0.891)$ & $(1698.81,0.5350)$ & 9 & 1.0123 & 0.1407 & 0.5156 \\
\hline 8C3SU3 & 8 & 3 & $(1111.52,0.1016)$ & $(1668.91,0.4988)$ & 5 & 0.9057 & 0.2040 & 0.6994 \\
\hline 8C3SU4 & 8 & 2 & $(1382.32,0.1192)$ & $(4004.33,0.4877)$ & 8 & 1.2267 & 0.1093 & 0.7305 \\
\hline 10C3SU1 & 10 & 5 & $(2183.75,0.0321)$ & $(11145.2,0.2237)$ & 11 & 0.8358 & 0.0624 & 0.7168 \\
\hline 10C3SU2 & 10 & 4 & $(2530.02,0.0327)$ & $(6283.93,0.2282)$ & 8 & 0.6955 & 0.0613 & 0.7052 \\
\hline 10C3SU3 & 10 & 4 & $(3574.33,0.1243)$ & $(19077.5,0.3288)$ & 9 & 1.2514 & 0.0983 & 0.6413 \\
\hline 10C3SU4 & 10 & 4 & $(2073.33,0.0303)$ & $(13827.4,0.3124)$ & 15 & 1.1712 & 0.0730 & 0.7198 \\
\hline $12 \mathrm{C} 3 \mathrm{SU} 1$ & 12 & 4 & $(2048.78,0.0530)$ & $(12189.5,0.2318)$ & 10 & 1.0227 & 0.0930 & 0.6892 \\
\hline $12 \mathrm{C} 3 \mathrm{SU} 2$ & 12 & 6 & $(3022.50,0.0637)$ & $(9649.21,0.2331)$ & 7 & 0.9543 & 0.1303 & 0.6049 \\
\hline $12 \mathrm{C} 3 \mathrm{SU} 3$ & 12 & 4 & $(6681.92,0.0688)$ & $(43126.9,0.2040)$ & 13 & 1.2263 & 0.1357 & 0.8525 \\
\hline $12 \mathrm{C} 3 \mathrm{SU} 4$ & 12 & 5 & $(5722.19,0.0307)$ & $(27551.8,0.2473)$ & 10 & 1.2884 & 0.1143 & 0.8061 \\
\hline 14C3SU1 & 14 & 6 & $(2006.7,0.0258)$ & $(8010.71,0.1257)$ & 8 & 0.5029 & 0.0466 & 0.8274 \\
\hline 14C3SU2 & 14 & 5 & $(2250.18,0.0227)$ & $(8683.59,0.1692)$ & 8 & 0.6992 & 0.0693 & 0.7011 \\
\hline 14C3SU3 & 14 & 6 & $(2396.2,0.0601)$ & $(6500.78,0.1325)$ & 8 & 0.7821 & 0.0626 & 0.7010 \\
\hline 14C3SU4 & 14 & 6 & $(1927.41,0.0366)$ & $(4799.84,0.1793)$ & 7 & 1.0831 & 0.1279 & 0.6613 \\
\hline 16C3SU1 & 16 & 5 & $(2165.25,0.0481)$ & $(8826.63,0.1350)$ & 8 & 1.1417 & 0.0910 & 0.8100 \\
\hline 16C3SU2 & 16 & 6 & $(3092.06,0.0505)$ & $(7934.97,0.0956)$ & 10 & 0.4095 & 0.0424 & 0.5190 \\
\hline 16C3SU3 & 16 & 6 & $(6961.15,0.0154)$ & $(28132.4,0.1392)$ & 9 & 1.3024 & 0.1380 & 0.7837 \\
\hline $16 \mathrm{C} 3 \mathrm{SU} 4$ & 16 & 6 & $(4820.75,0.0143)$ & $(13715.5,0.0896)$ & 7 & 0.6999 & 0.0906 & 0.815 \\
\hline
\end{tabular}

\section{Conclusions and future research}

This paper has presented the bi-objective green vehicle routing problem with alternative paths wherein each path has its specific reliability whereas the length of each alternative route also varies. For the first objective function, the costs are minimized, and the second objective function is to maximize the reliability of the whole system. This model was presented as nonlinear mixed-integer programming. By considering the combination of green vehicle routing problem and reliability that each of which is recognized as NP-hard, the complexity of this problem was further increased. Therefore, it was almost impossible to apply an exact solution to large dimensions. In this regard, MOSA's meta-heuristic algorithm has been used to solve the problem. MOSA's results were compared with the epsilon constraint's results to evaluate the quality of them. To evaluate the quality of the obtained results, performance metrics as the number of non-dominated solutions, maximum spread, spacing, and mean ideal distance were used. The results show the efficiency of the proposed method in producing nearoptimal responses at the proper execution time. By increasing costs and using more reliable paths, system reliability rises, which has been shown in the Pareto fronts. In today's competitive world, where companies are looking for higher services' quality, system reliability is essential. This article helps decision-makers choose the desired answer according to the considered level of reliability and acceptable cost. 
Further research in this field could include: Considering uncertainties and probable and fuzzy parameters; Considering traffic zones where the use of more polluting fuels is prohibited; Adding familiar complementary elements to the standard vehicle routing problem such as multiple warehouses, time windows, heterogeneous fleets, etc.; Considering electric vehicles and their unique features; Using multi-objective meta-heuristic algorithms such as NSGA-II and MOPSO to compare the performance of the MOSA algorithm and improve problem-solving especially in large sizes; Implementation of the proposed mathematical model in a real case study.

\section{Acknowledgement}

The authors would like to thank the anonymous referees for constructive comments on earlier version of this paper.

\section{References}

Adenso-Diaz, B., Mena, C., García-Carbajal, S., \& Liechty, M. (2012). The impact of supply network characteristics on reliability. Supply Chain Management: An International Journal, 17(3), 263-276.

Affi, M., Derbel, H., \& Jarboui, B. (2018). Variable neighborhood search algorithm for the green vehicle routing problem. International Journal of Industrial Engineering Computations, 9(2), 195204.

Avila, C., \& Valdez, F. (2015). An improved simulated annealing algorithm for the optimization of mathematical functions. In Design of Intelligent Systems Based on Fuzzy Logic, Neural Networks and Nature-Inspired Optimization (pp. 241-251). Springer, Cham.

Baños, R., Ortega, J., Gil, C., MáRquez, A. L., \& De Toro, F. (2013). A hybrid meta-heuristic for multiobjective vehicle routing problems with time windows. Computers \& Industrial Engineering, 65(2), 286-296.

Beamon, B. M. (1998). Supply chain design and analysis:: Models and methods. International journal of production economics, 55(3), 281-294.

Bektaş, T., \& Laporte, G. (2011). The pollution-routing problem. Transportation Research Part B: Methodological, 45(8), 1232-1250.

Bérubé, J. F., Gendreau, M., \& Potvin, J. Y. (2009). An exact $\epsilon$-constraint method for bi-objective combinatorial optimization problems: Application to the Traveling Salesman Problem with Profits. European journal of operational research, 194(1), 39-50.

Bruglieri, M., Pezzella, F., Pisacane, O., \& Suraci, S. (2015). A variable neighborhood search branching for the electric vehicle routing problem with time windows. Electronic Notes in Discrete Mathematics, 47, 221-228.

Castillo, O., Neyoy, H., Soria, J., Melin, P., \& Valdez, F. (2015). A new approach for dynamic fuzzy logic parameter tuning in ant colony optimization and its application in fuzzy control of a mobile robot. Applied Soft Computing, 28, 150-159.

Chávez, J., Escobar, J \& Echeverri, M. (2016). A multi-objective Pareto ant colony algorithm for the Multi-Depot Vehicle Routing problem with Backhauls. International Journal of Industrial Engineering Computations , 7(1), 35-48.

Chen, P., Huang, H. K., \& Dong, X. Y. (2010). Iterated variable neighborhood descent algorithm for the capacitated vehicle routing problem. Expert Systems with Applications, 37(2), 1620-1627.

Ćirović, G., Pamučar, D., \& Božanić, D. (2014). Green logistic vehicle routing problem: Routing light delivery vehicles in urban areas using a neuro-fuzzy model. Expert Systems with Applications, 41(9), 4245-4258.

Conrad, R. G., \& Figliozzi, M. A. (2011). The recharging vehicle routing problem. In Proceedings of the 2011 industrial engineering research conference (p. 8). IISE Norcross, GA.

Cordeau, J. F., \& Maischberger, M. (2012). A parallel iterated tabu search heuristic for vehicle routing problems. Computers \& Operations Research, 39(9), 2033-2050. 
Dekker, R., Bloemhof, J., \& Mallidis, I. (2012). Operations Research for green logistics-An overview of aspects, issues, contributions and challenges. European Journal of Operational Research, 219(3), 671-679.

Demir, E., Bektaş, T., \& Laporte, G. (2012). An adaptive large neighborhood search heuristic for the pollution-routing problem. European Journal of Operational Research, 223(2), 346-359.

Demir, E., Bektaş, T., \& Laporte, G. (2014). The bi-objective pollution-routing problem. European Journal of Operational Research, 232(3), 464-478.

Dorigo, M., \& Stützle, T. (2003). The ant colony optimization metaheuristic: Algorithms, applications, and advances. In Handbook of metaheuristics (pp. 250-285). Springer, Boston, MA.

Eberhart, R., \& Kennedy, J. (1995, October). A new optimizer using particle swarm theory. In MHS'95. Proceedings of the Sixth International Symposium on Micro Machine and Human Science (pp. 3943). IEEE.

Ehrgott, M., \& Gandibleux, X. (2003). Multiobjective combinatorial optimization-theory, methodology, and applications. In Multiple criteria optimization: State of the art annotated bibliographic surveys (pp. 369-444). Springer, Boston, MA.

Erdoğan, S., \& Miller-Hooks, E. (2012). A green vehicle routing problem. Transportation Research Part E: Logistics and Transportation Review, 48(1), 100-114.

Farahani, R.Z., Rezapour, S., \& Kardar, L. (2011). Logistics operations and management: concepts and models. Elsevier.

Felipe, Á., Ortuño, M. T., Righini, G., \& Tirado, G. (2014). A heuristic approach for the green vehicle routing problem with multiple technologies and partial recharges. Transportation Research Part E: Logistics and Transportation Review, 71, 111-128.

Franceschetti, A., Honhon, D., Van Woensel, T., Bektaş, T., \& Laporte, G. (2013). The time-dependent pollution-routing problem. Transportation Research Part B: Methodological, 56, 265-293.

Garaix, T., Artigues, C., Feillet, D., \& Josselin, D. (2010). Vehicle routing problems with alternative paths: An application to on-demand transportation. European Journal of Operational Research, 204(1), 62-75.

Garcia-Najera, A., \& Bullinaria, J. A. (2011). An improved multi-objective evolutionary algorithm for the vehicle routing problem with time windows. Computers \& Operations Research, 38(1), $287-$ 300 .

Gendreau, M., Laporte, G., \& Potvin, J. Y. (2002). Metaheuristics for the capacitated VRP. In The vehicle routing problem (pp. 129-154). Society for Industrial and Applied Mathematics.

Glover, F. (1999). Scatter search and path relinking. New ideas in optimization, 297316.

Glover, F., \& Laguna, M. (2013). Tabu search: effective strategies for hard problems in analytics and computational science. Handbook of Combinatorial Optimization, 21, 3261-3362.

Goldberg, D. E. (1989), Genetic Algorithms in Search, Optimization and Machine Learning, AddisonWesley, New York.

Gonçalves, F., Cardoso, S. R., Relvas, S., \& Barbosa-Póvoa, A. P. F. D. (2011). Optimization of a distribution network using electric vehicles: A VRP problem. In Proceedings of the IO2011-15 Congresso da associação Portuguesa de Investigação Operacional, Coimbra, Portugal (pp. 18-20).

Ha, C., Jun, H. B., \& Ok, C. (2018). A mathematical definition and basic structures for supply chain reliability: A procurement capability perspective. Computers \& Industrial Engineering, 120, 334345.

Holland, J. H. (1975). Adaptation in Natural and Artificial Systems. Ann Arbor, Michigan, USA: The University of Michigan Press.

Jozefowiez, N., Semet, F., \& Talbi, E. G. (2008). Multi-objective vehicle routing problems. European journal of operational research, 189(2), 293-309.

Jozefowiez, N., Semet, F., \& Talbi, E. G. (2009). An evolutionary algorithm for the vehicle routing problem with route balancing. European Journal of Operational Research, 195(3), 761-769.

Kara, I., Kara, B. Y., \& Yetis, M. K. (2007, August). Energy minimizing vehicle routing problem. In International Conference on Combinatorial Optimization and Applications(pp. 62-71). Springer, Berlin, Heidelberg. 
Kirkpatrick, S., Gelatt, C. D., \& Vecchi, M. P. (1983). Optimization by simulated annealing. Science, 220(4598), 671-680.

Klimov, R., \& Merkuryev, Y. (2008). Simulation model for supply chain reliability evaluation. Technological and Economic Development of Economy, 14(3), 300-311.

Kuo, Y. (2010). Using simulated annealing to minimize fuel consumption for the time-dependent vehicle routing problem. Computers \& Industrial Engineering, 59(1), 157-165.

Kytöjoki, J., Nuortio, T., Bräysy, O., \& Gendreau, M. (2007). An efficient variable neighborhood search heuristic for very large scale vehicle routing problems. Computers \& Operations Research, 34(9), 2743-2757.

Laporte, G. (2009). Fifty years of vehicle routing. Transportation Science, 43(4), 408-416.

Lin, C., Choy, K. L., Ho, G. T., Chung, S. H., \& Lam, H. Y. (2014). Survey of green vehicle routing problem: past and future trends. Expert Systems With Applications, 41(4), 1118-1138.

Lin, S. W., \& Vincent, F. Y. (2012). A simulated annealing heuristic for the team orienteering problem with time windows. European Journal of Operational Research, 217(1), 94-107.

Lin, S. W., Lee, Z. J., Ying, K. C., \& Lee, C. Y. (2009). Applying hybrid meta-heuristics for capacitated vehicle routing problem. Expert Systems with Applications, 36(2), 1505-1512.

Lin, S. W., Vincent, F. Y., \& Lu, C. C. (2011). A simulated annealing heuristic for the truck and trailer routing problem with time windows. Expert Systems with Applications, 38(12), 15244-15252.

Liu, S., So, K. C., \& Zhang, F. (2010). Effect of supply reliability in a retail setting with joint marketing and inventory decisions. Manufacturing \& Service Operations Management, 12(1), 19-32.

Lourenço, H. R., Martin, O. C., \& Stützle, T. (2003). Iterated local search. In Handbook of metaheuristics (pp. 320-353). Springer, Boston, MA.

Mancini, S. (2017). The hybrid vehicle routing problem. Transportation Research Part C: Emerging Technologies, 78, 1-12.

Metropolis, N., Rosenbluth, A. W., Rosenbluth, M. N., Teller, A. H., \& Teller, E. (1953). Equation of state calculations by fast computing machines. The Journal of Chemical Physics, 21(6), 1087-1092.

Mitra, D., Romeo, F., \& Sangiovanni-Vincentelli, A. (1986). Convergence and finite-time behavior of simulated annealing. Advances in Applied Probability, 18(3), 747-771.

Mladenović, N., \& Hansen, P. (1997). Variable neighborhood search. Computers \& Operations Research, 24(11), 1097-1100.

Moscato, P. (1989). On Evolution, Search, Optimization, Genetic Algorithms and Martial Arts: Towards Memetic Algorithms. Caltech Concurrent Computation Program.

Moscato, P., \& Cotta, C. (2010). A modern introduction to memetic algorithms. In Handbook of metaheuristics (pp. 141-183). Springer, Boston, MA.

Nam, D., \& Park, C. H. (2000). Multiobjective simulated annealing: A comparative study to evolutionary algorithms. International Journal of Fuzzy Systems, 2(2), 87-97.

Nishiguchi, T. \& Beaudet, A., (1998). The Toyota Group and the Aisin Fire. Sloan Management Review, 40, 49-59.

Norouzi, N., Tavakkoli-Moghaddam, R., Salamatbakhsh, A., \& Alinaghian, M. (2009). Solving a novel bi-objective open vehicle routing problem in a competitive situation by multi objective particle swarm optimization. Journal of Applied Operations Research, 1(1), 15-29.

Ombuki, B., Ross, B. J., \& Hanshar, F. (2006). Multi-objective genetic algorithms for vehicle routing problem with time windows. Applied Intelligence, 24(1), 17-30.

Osman, I. H. (1993). Metastrategy simulated annealing and tabu search algorithms for the vehicle routing problem. Annals of Operations Research, 41(4), 421-451.

Ping, L. (2009). Strategy of green logistics and sustainable development. In 2009 International Conference on Information Management, Innovation Management and Industrial Engineering (Vol. 1, pp. 339-342). IEEE.

Poli, R., Kennedy, J., \& Blackwell, T. (2007). Particle swarm optimization. Swarm Intelligence, 1(1), 33-57. 
Reed, B. D., Smas, M. J., Rzepka, R. A., \& Guiffrida, A. L. (2010). Introducing green transportation costs in supply chain modeling. In Proceedings of the First Annual Kent State International Symposium on Green Supply Chains (pp. 189-197).

Resende, M. G., Ribeiro, C. C., Glover, F., \& Martí, R. (2010). Scatter search and path-relinking: Fundamentals, advances, and applications. In Handbook of metaheuristics (pp. 87-107). Springer, Boston, MA.

Rincon-Garcia, N., Waterson, B., \& Cherrett, T. (2017). A hybrid metaheuristic for the time-dependent vehicle routing problem with hard time windows. International Journal of Industrial Engineering Computations, 8(1), 141-160.

Rochat, Y., \& Taillard, É. D. (1995). Probabilistic diversification and intensification in local search for vehicle routing. Journal of Heuristics, 1(1), 147-167.

Ropke, S., \& Pisinger, D. (2006). An adaptive large neighborhood search heuristic for the pickup and delivery problem with time windows. Transportation Science, 40(4), 455-472.

Schneider, M., Stenger, A., \& Goeke, D. (2014). The electric vehicle-routing problem with time windows and recharging stations. Transportation Science, 48(4), 500-520.

Schott, J. R. (1995). Fault Tolerant Design Using Single and Multi-Criteria Genetic Algorithms. Master's Thesis, Department of Aeronautics and Astronautics, Massachusetts Institute of Technology.

Serafini, P. (1994). Simulated annealing for multi objective optimization problems. In Multiple criteria decision making (pp. 283-292). Springer, New York, NY.

Taillard, É. (1993). Parallel iterative search methods for vehicle routing problems. Networks, 23(8), 661-673.

Tan, K. C., Chew, Y. H., \& Lee, L. H. (2006). A hybrid multiobjective evolutionary algorithm for solving vehicle routing problem with time windows. Computational Optimization and Applications, 34(1), 115.

Tiwari, A., \& Chang, P. C. (2015). A block recombination approach to solve green vehicle routing problem. International Journal of Production Economics, 164, 379-387.

Tseng, Y. Y., Yue, W. L., \& Taylor, M. A. (2005). The role of transportation in logistics chain. Eastern Asia Society for Transportation Studies.

Ulungu, E. L., Teghem, J. F. P. H., Fortemps, P. H., \& Tuyttens, D. (1999). MOSA method: a tool for solving multiobjective combinatorial optimization problems. Journal of Multi-Criteria Decision Analysis, 8(4), 221-236.

Vidal, T., Crainic, T. G., Gendreau, M., \& Prins, C. (2013). Heuristics for multi-attribute vehicle routing problems: A survey and synthesis. European Journal of Operational Research, 231(1), 121.

Vincent, F. Y., Jewpanya, P., \& Redi, A. P. (2016). Open vehicle routing problem with crossdocking. Computers \& Industrial Engineering, 94, 6-17.

Vincent, F. Y., Lin, S. W., Lee, W., \& Ting, C. J. (2010). A simulated annealing heuristic for the capacitated location routing problem. Computers \& Industrial Engineering, 58(2), 288-299.

Vincent, F. Y., Redi, A. P., Hidayat, Y. A., \& Wibowo, O. J. (2017). A simulated annealing heuristic for the hybrid vehicle routing problem. Applied Soft Computing, 53, 119-132.

Xiao, Y., Zhao, Q., Kaku, I., \& Xu, Y. (2012). Development of a fuel consumption optimization model for the capacitated vehicle routing problem. Computers \& Operations Research, 39(7), 1419-1431.

Zaitsev, E. (2012). Supply chain reliability modelling. LogForum, 8(1), 61-69.

Zitzler, E. (1999). Evolutionary algorithms for multiobjective optimization: Methods and applications (Vol. 63). Ithaca: Shaker. 


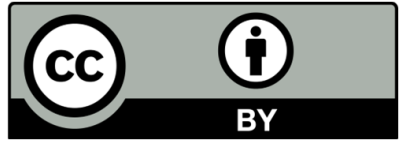

(C) 2020 by the authors; licensee Growing Science, Canada. This is an open access article distributed under the terms and conditions of the Creative Commons Attribution (CC-BY) license (http://creativecommons.org/licenses/by/4.0/). 